\title{
CORRECTION
}

\section{Correction to: Assessment of Bacterial Colonization of Intracranial Pressure Transducers: A Prospective Study}

Roman Mounier ${ }^{1,2^{*}}$, Natacha Kapandji', Guillaume Gricourt ${ }^{3}$, David Lobo ${ }^{1}$, Christophe Rodriguez ${ }^{3}$, Stéphanie Pons ${ }^{1}$, Chakib Djediat ${ }^{4}$, Paul-Louis Woerther ${ }^{5}$, Vincent Mellano ${ }^{1}$, Bouziane Aït-Mamar', Vanessa Demontant ${ }^{3}$, Biba Nebbad ${ }^{4}$, Suhan Senova ${ }^{2,6}$, Melissa Arnaud ${ }^{1}$, Fabrice Cook ${ }^{1}$, Gilles Dhonneur ${ }^{1}$ and David Lebeaux ${ }^{7,8}$

(C) 2020 Springer Science+Business Media, LLC, part of Springer Nature and Neurocritical Care Society

\section{Correction to: Neurocrit Care \\ https://doi.org/10.1007/s12028-020-01096-x}

The original article was updated to correct the co-author name Suhan Senova in author list. The details mentioned here are correct.

\section{Author details}

${ }^{1}$ Department of Anesthesia and Surgical Intensive Care, Henri Mondor University Hospital of Paris, Paris-Est Créteil University, 51, Avenue du Maréchal de Lattre de Tassigny, 94000 Créteil, France. ${ }^{2}$ INSERM U955, team 15, MondorInstitute for Biomedical Research, University Paris-Est-Créteil (UPEC), Créteil, France. ${ }^{3}$ Department of Microbiology, Platform NGS Henri Mondor University Hospital of Paris, Institut Mondor de la Recherche Biomédicale, Paris-Est
Créteil University, 94000 Créteil, France. ${ }^{4}$ Plateau Technique de Microscopie Électronique, MET, UMR7245, Département AVIV, Museum National d'Histoire Naturelle, Paris, France. ${ }^{5}$ Department of Microbiology, Henri Mondor University Hospital Assitance Publique-Hôpitaux de Paris (APHP), University Paris-Est-Créteil (UPEC), Paris, France. ${ }^{6}$ Department of Neurosurgery, Henri Mondor University Hospital of Paris, Paris XII School of Medicine, 94000 Créteil, France. ${ }^{7}$ Université de Paris, 75006 Paris, France. ${ }^{8}$ Service de Microbiologie, Unité Mobile D'Infectiologie, AP-HP, Hôpital Européen Georges Pompidou, 20 rue Leblanc, 75015 Paris, France.

\section{Publisher's Note}

Springer Nature remains neutral with regard to jurisdictional claims in published maps and institutional affiliations.

Published online: 15 October 2020 